\title{
Editorial
}

\section{The powerful potential of low-cost sensors for air quality research in Africa}

\author{
R Subramanian (1) ${ }^{1^{*}}$ and Rebecca M Garland (1) ${ }^{2}$ \\ ${ }^{1}$ Kigali Collaborative Research Centre, Kigali, Rwanda. Currently at \\ Qatar Environment and Energy Research Institute, Hamad Bin Khalifa University, Doha, Qatar \\ ${ }^{2}$ Climate and Air Quality Modelling Research Group, CSIR, South Africa \\ *Dr Subramanian is a guest editor for this special feature on low-cost sensors for air quality research.
}

https://doi.org/10.17159/caj/2021/31/1.11274

\begin{abstract}
Low-cost sensors (LCS) are becoming popular with air quality stakeholders all over the world, including researchers, policymakers, and citizen scientists. They are increasingly being used in research where air quality data are sparse, such as in Africa. This featured commentary section showcases three thoughtful, considered efforts, where LCS devices are carefully evaluated against reference monitors and corrected to ensure high data quality.
\end{abstract}

These three commentaries highlight studies where LCS are being used for higher spatial and temporal resolution monitoring for later comparison with satellite data and models (Giordano and Jaramillo, 2021), for public awareness and stakeholder engagement for pollution mitigation (Sewor et al. 2021), and epidemiological studies and investigating household air pollution (Wernecke et al. 2021). The efforts highlighted here are focused on measuring ambient particulate matter mass concentrations (PM); however, it is important to note that other pollutants, especially ozone $\left(\mathrm{O}_{3}\right)$ and nitrogen dioxide $\left(\mathrm{NO}_{2}\right)$, are also major environmental concerns. More effort is needed to increase the monitoring of those pollutants using both LCS and reference monitors.

In addition to highlighting the potential of LCS for air quality research, these contributions reveal some important challenges. LCS data completion can be affected by power outages and WiFi connectivity. The former can be mitigated by the use of solar powered sensors. Data connectivity can be solved with general packet radio service (GPRS), narrow-band internet of things (NBIoT), and similar wireless networks, but these options may be expensive and local laws may not be loT-friendly.

Commercial sensors like PurpleAir and Clarity may be low-cost for many users, but they are still too expensive for scientists in many African countries. Opportunities for local development, such as those by AirQo and Code for Africa, should be supported in order to reduce costs and ensure compatibility with local climates.

Currently, there are no standards or certification for low-cost sensors; standardized testing conducted by American or European bodies (e.g. the South Coast Air Quality Management District's Air Quality Sensor Performance Evaluation Center, AQ-SPEC) may not reflect performance in African environments, pollution levels, and PM composition. Hence, there is a need for instrument intercomparisons and for local testing centers. Wernecke et al. (2021) describes such an effort at the North-West University in South
Africa. The need and potential for similar facilities in other regions and environments (e.g. equatorial, desert) should be explored and linked to form a collaborative network of such facilities.

We strongly encourage data transparency that empowers citizens to consider personal changes and support systemic efforts to reduce their exposure to air pollution; the power of LCS networks is only truly unleashed with open data (Sewor et al. 2021). National air quality monitoring data are publicly available from only a few countries across Africa, such as South Africa and Rwanda. The US Department of State has installed near-reference air quality monitors at twelve embassies across Africa, and the data are available in near real-time. Many LCS datasets are also available through international repositories like OpenAQ or manufacturer websites - but only when the sensor owner chooses to share such data. One concern for LCS data sets is whether appropriate corrections have been applied to such openly-shared data; the data are not useful for research if uncorrected. Nevertheless, data transparency is increasingly the norm, and we strongly encourage researchers using LCS to share their corrected data. Giordano and Jaramillo (2021) describe a nuanced approach taking into account the need to recognize the efforts put into collecting such data while ensuring data transparency.

These three commentaries highlight some of the work ongoing on this issue in Africa. The Clean Air Journal welcomes submissions on all aspects of this topic including sensor development, sensor calibration and performance, monitoring data, air pollution analysis, and exposure studies using carefully-calibrated low-cost sensors.

\section{References}

Giordano, M.R. and Jaramillo, P. 2021 AfriqAir's mission towards cleaner air for Africa and a call for action. Clean Air Journal, 31(1) doi: $10.17159 / \mathrm{caj} / 2021 / 31 / 1.11173$

Sewor, C., Obeng, A.A., Amegah, A.K. 2021. The Ghana Urban Air Quality Project (GHAir): Bridging air pollution data gaps in Ghana. Clean Air Journal, 31(1) doi: 10.17159/caj/2021/31/1.11172

Wernecke, B. et al. 2021. Opportunities for the application of lowcost sensors in epidemiological studies to advance evidence of air pollution impacts on human health. Clean Air Journal, 31(1), doi: 10.17159/caj/2021/31/1.11219. 